\title{
ElncRNA1, a long non-coding RNA that is transcriptionally induced by oestrogen, promotes epithelial ovarian cancer cell proliferation
}

\author{
JUN-JUN QIU ${ }^{1-3}$, XIAO-DAN ZHANG ${ }^{1-3}$, XIAO-YAN TANG ${ }^{1-3}$, \\ TING-TING ZHENG ${ }^{1-3}$, YING ZHANG ${ }^{1-3}$ and KE-QIN HUA ${ }^{1-3}$
}

\begin{abstract}
${ }^{1}$ Department of Gynecology, Obstetrics and Gynecology Hospital of Fudan University, Shanghai 200011; ${ }^{2}$ Department of Obstetrics and Gynecology of Shanghai Medical College, Fudan University, Shanghai 200032; ${ }^{3}$ Shanghai Key Laboratory of Female Reproductive Endocrine-Related Diseases, Shanghai 200011, P.R. China
\end{abstract}

Received December 6, 2016; Accepted April 7, 2017

DOI: 10.3892/ijo.2017.4030

\begin{abstract}
Previously, the novel oestrogen (E2)-upregulated IncRNA TC0101441, was identified by us, via microarray analysis. However, the detailed mechanism by which E2 upregulates TC0101441 and the role of TC0101441 in epithelial ovarian cancer (EOC) progression have not been elucidated. In the present study, we further analysed TC0101441, which we designated oestrogen-induced long non-coding RNA-1 (ElncRNA1). We showed that E2 transcriptionally upregulates ElncRNA1 through the oestrogen receptor $\alpha(E R \alpha)$-oestrogen response element (ERE) pathway using RNA stability assays, bioinformatics-based searches for ERE binding sites, chromatin immunoprecipitation (ChIP) assays and dual luciferase reporter assays. Clinically, ElncRNA1 levels are significantly higher in EOC tissues than in normal ovarian surface epithelium. In vitro and in vivo loss-of-function assays revealed that ElncRNA1 promotes EOC cell proliferation. This pro-proliferation effect of ElncRNA1 was partially mediated by the regulation of CDK4, CDK6 and cyclin D1. These findings provide the first evidence that E2 upregulates ElncRNA1 at the transcriptional level through the ER $\alpha$-ERE pathway and that this novel E2-upregulated lncRNA has an oncogenic role in EOC growth. The placement of ElncRNA1 in the E2-ER $\alpha$-ERE signalling pathway may provide greater insight into the effects of oestrogen on EOC progression from the perspective of lncRNA.
\end{abstract}

\section{Introduction}

Epithelial ovarian cancer (EOC) is the leading cause of death from gynaecologic malignancies (1-3). EOC is highly lethal,

Correspondence to: Dr Ke-qin Hua, Department of Gynecology, Obstetrics and Gynecology Hospital of Fudan University, 419 Fangxie Road, Shanghai 200011, P.R. China

E-mail: huakeqin@126.com

Key words: long non-coding RNA, ElncRNA1, oestrogen, ovarian cancer, proliferation primarily because it is diagnosed at an advanced stage and is characterized by aggressive proliferation and metastasis (2). Despite advances in therapy, the high mortality and poor prognosis associated with EOC remain unchanged (2). Therefore, identifying new therapeutic targets for EOC and understanding the molecular mechanisms underlying EOC aggressiveness will yield novel strategies for overcoming this malignancy.

Accumulating clinical and epidemiological evidence has shown that oestrogen (E2) is responsible for promoting ovarian cancer progression (4,5). In vitro and in vivo experimental data have indicated that E2 enhances EOC cell proliferation (6-11). The effects of E2 on EOC proliferation are mainly attributable to E2-regulated target genes. Previous studies have reported numerous E2-regulated proteins that affect EOC cell proliferation and progression, such as cyclin D1, c-myc and insulin-like growth factor-binding protein (IGFBP) family members $(5,12)$. Despite the identification of these E2-regulated proteins, the exact effect of E2 on EOC progression is not fully understood. Therefore, there is an urgent need to identify novel E2-regulated target genes.

The discovery of long non-coding RNAs (lncRNAs, $>200$ nucleotides) has opened a new avenue in cancer research. Accumulating evidence highlights the importance of lncRNAs as a novel class of oncogenes or tumour suppressor genes in the development of many types of cancer (13-23), including EOC (24-28). Further, some lncRNAs have recently been reported to be involved in E2 signalling. For example, the lncRNA-SRA1 was reported to function as a nuclear receptor corepressor in E2 signalling (29), and HOTAIR is upregulated by E2 and contributes to E2-supported breast cancer progression $(30,31)$. However, little is known about E2-regulated lncRNAs in ovarian cancer.

We previously identified a novel E2-upregulated lncRNA, TC0101441, based on microarray analysis and found that TC0101441 contributes to E2-induced EOC cell migration (32). However, the detailed mechanism by which E2 upregulates TC0101441 and the role of ElncRNA1 itself in EOC progression have not been elucidated. In the present study, we further evaluated TC0101441, designated as oestrogen-induced long non-coding RNA-1 (ElncRNA1), and showed that E2 
upregulated ElncRNA1 at the transcriptional level through the oestrogen receptor $\alpha(E R \alpha)$-oestrogen response element (ERE) pathway. Clinically, ElncRNA1 levels were significantly higher in EOC tissues than in normal ovarian surface epithelial tissues. In vitro and in vivo assays revealed that ElncRNA1 promotes EOC cell proliferation. This pro-proliferation effect of ElncRNA1 was partially mediated by the regulation of CDK4, CDK6 and cyclin D1. These findings not only clarify the mechanism by which E2 upregulates ElncRNA1 but also underscore the important role of this novel E2-upregulated lncRNA in EOC proliferation, thus providing a connection between E2 and ovarian cancer from the perspective of lncRNA.

\section{Materials and methods}

Cell lines and clinical tissue samples. The human EOC cell lines (SKOV3, CAOV3, OVCAR3 and HO8910) were a gift from University of Texas MD Anderson Cancer Center (Houston, TX, USA). SKOV3, CAOV3 and HO8910 cells were cultured in RPMI-1640 (Gibco, Gaithersburg, MD, USA). OVCAR3 cells were maintained in McCoy's 5A supplemented with $10 \%$ foetal bovine serum (Gibco) at $37^{\circ} \mathrm{C}$ in a $5 \% \mathrm{CO}_{2}$ atmosphere. For E2 treatment, cells (plated at 20-30\% confluence) were grown for 3 days in phenol red-free RPMI-1640 or McCoy's 5A containing 5\% charcoal-stripped foetal bovine serum (Serana, Bunbury, Australia). Next, the cells were treated for $24 \mathrm{~h}$ with $10^{-8} \mathrm{M} \mathrm{E2} \mathrm{(Sigma)} \mathrm{or} \mathrm{vehicle} \mathrm{(DMSO,}$ $0.01 \%$ of final volume) as a control.

All tissue samples were obtained during surgical operations at the Obstetrics and Gynecology Hospital of Fudan University between August 2005 and December 2008. All EOC tissues were selected from patients who a) had not received preoperative radiotherapy, chemotherapy, or hormonal therapy; and b) did not have borderline ovarian tumours or two or more different malignancies. All 40 EOC tissues analysed herein were serous ovarian cancer tissues. In particular, according to the International Federation of Gynecologists and Obstetricians (FIGO) staging system, 6 cases were FIGO stage I, 6 cases were FIGO stage II, 24 cases were FIGO stage III, and 4 cases were FIGO stage IV. Additionally, according to histological grading guidelines, 6 cases were grade I (G1), 12 cases were G2, and 22 cases were G3. The normal ovarian epithelial tissues were obtained from participants diagnosed with uterine fibroids who were scheduled to undergo a hysterectomy and oophorectomy. Tissues were selected from participants who did not have a history of ovarian cysts, ovarian pathology, or ovarian surgery. All samples were pathologically confirmed, immediately frozen in liquid nitrogen and stored at $-80^{\circ} \mathrm{C}$. The study was approved by the Research Ethics Committee of Fudan University, China. Informed consent was obtained from each patient.

RNA extraction and quantitative real-time polymerase chain reaction ( $q R T-P C R)$. Total RNA was isolated with TRIzol reagent (Invitrogen, Carlsbad, CA, USA) and then reverse transcribed into cDNA using the ExScript RT-PCR kit (Takara, Otsu, Japan) following the manufacturer's instructions. ElncRNA1 expression was measured by qRT-PCR using the following primer sequences: forward, 5'-CAAGGCAGGTGA GAACGAGT-3'; and reverse, 5'-CTCGACTTAGGGAGCT
GCAC-3'. Expression of the internal control, glyceraldehyde 3-phosphate dehydrogenase (GAPDH), was measured with the following primers: forward, 5'-TGACTTCAACAGCGA CACCCA-3'; and reverse, 5'-CACCCTGTTGCTGTAGCC AAA-3'. Amplification and detection were conducted using a Prism 7900 system (Applied Biosystems, Foster City, CA, USA) with the ExScript SYBR Green qRT-PCR kit (Takara). All assays were repeated in triplicate, and statistical analyses of the results were performed using the $2^{-\Delta \Delta \mathrm{Ct}}$ relative quantification method.

Sequence analysis of ElncRNA1. The gene location was obtained from the University of California Santa Cruz (UCSC) genome browser (hg18, http://genome.ucsc.edu/). The Basic Local Alignment Search Tool (BLAST) was used for sequence alignment, and the BLAST-Like Alignment Tool (BLAT) was used to map the cDNA to chromosomes.

RNA stability assay. To measure RNA half-life, we added $10 \mu \mathrm{g} / \mathrm{ml}$ actinomycin D (Sigma) to the cells to block transcription (nascent RNA synthesis). The cells were pre-treated with actinomycin D prior to E2 treatment. Total RNA was extracted from samples harvested at the indicated time points. ElncRNA1 expression was quantified by qRT-PCR. The halflife of ElncRNA1 RNA was calculated using data from two independent experiments.

Search for EREs. A region 2,500 bp upstream and 500 bp downstream of the transcriptional start site (TSS) was screened for transcription factor binding sites (TFBSs) using the Patser program (http://ural.wustl.edu/src/patser-v3e.1.tar. gz) and TRANSFAC 8.1 position weight matrices (PWMs) (http://www.gene-regulation.com/). A cut-off score of 0.9 was employed, and both the forward and reverse genomic DNA strands were investigated. EMBOSS/fuzznuc software (http:// helixweb.nih.gov/emboss/html/fuzznuc.html) was used to evaluate the putative EREs in the E2-regulated lncRNA. The conserved ERE sequence is AGGTCAnnnTGACCT, and up to two mismatches were allowed.

Plasmids and luciferase assays. A firefly luciferase (Luc) reporter driven by a fragment of the 3' untranslated regions (UTR) of ElncRNA1 containing the putative ERE [Luc-ElncRNA1-3'UTR wild-type (WT)] and a Luc reporter driven by a corresponding mutant sequence (Luc-ElncRNA13'UTR Mut) were synthesized. The primer sequences were as follows: sense for Luc-ElncRNA1-3'UTR WT and Luc-ElncRNA1-3'UTR Mut: GGGGTACCGTAGAGACAGG GTCTCACCACATT; antisense for Luc-ElncRNA1-3'UTR WT: CGACGCGTGGAGCATCTGTCCCAGCTG; and antisense for Luc-ElncRNA1-3'UTR Mut: CGACGCGTGGAGC ATCTGTCCCAGCTGGAGTCCCCTGTGGTGTTATCTG AGATtCAGCAGCTCTGCAAGAATATCATCTATGAC. A plasmid expressing ER $\alpha$ was constructed by Hanyin Co. (Shanghai, China). A plasmid expressing Renilla Luc was purchased from Promega (Madison, WI, USA). SKOV3 cells were transfected with the above plasmids or vectors for $24 \mathrm{~h}$ and then treated with E2; Luc assays were performed after a $16 \mathrm{~h}$ E2 treatment using a Dual-Luciferase Assay kit (Promega) according to the manufacturer's protocol. 


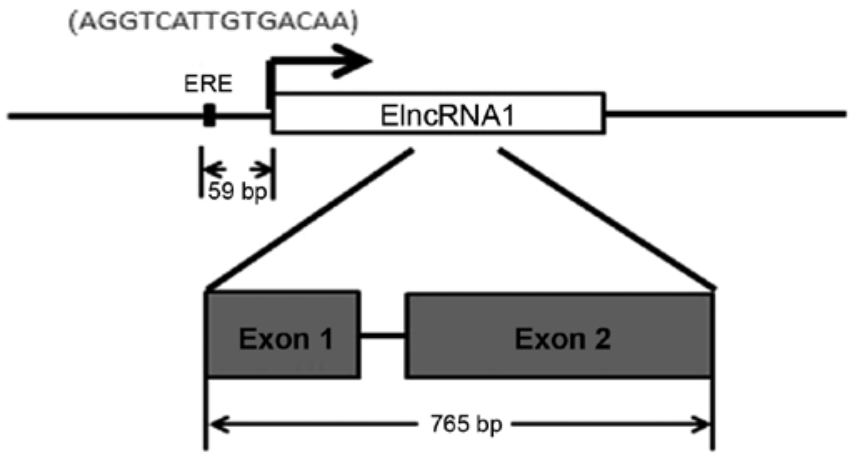

Figure 1. Characterization of the ElncRNA1 transcript. Diagram of the ElncRNA1 gene locus. The ElncRNA1 gene consists of 2 exons. The predicted ERE is located in the 59 bp region upstream of the TSS of ElncRNA1.

Chromatin immunoprecipitation (ChIP). ChIP assays were performed using a ChIP assay kit (Millipore) according to the manufacturer's instruction. Specific antibodies were used to immunoprecipitate either ER $\alpha$ or the negative control IgG. Real-time PCR was performed using a SYBR Green PCR kit (Qiagen). The primer sequences for amplifying the ElncRNA1 promoter region flanking the ER $\alpha$-ERE binding sites were as follows: forward, 5'-GGAAGAACAGCTCCGTGAAG-3'; and reverse, 5'-CAGATTCAGGGCTCTTGAGG-3'.

Establishment of stable ElncRNA1-knockdown cell lines. The two ElncRNA1-siRNA sequences were 5'-GCTCACATGA GAAAGCAAACT-3' (siRNA1) and 5'-CUUGAGUUAUG AGGUAGCA-3' (siRNA2). Lentiviral vectors encoding ElncRNA1-shRNA were designed based on the two siRNA sequences (ElncRNA1-knockdown (KD) 1 and ElncRNA1-KD2) and were constructed by Hanyin Co. The recombinant lentiviruses (KD1 and KD2) and the negative control (NC) lentivirus (Hanyin Co.) were prepared at a titre of $10^{9}$ transfection units (TU)/ml. To obtain stable cell lines, SKOV3 and CAOV3 cells were seeded in 6-well plates and infected with virus and polybrene the following day. Positive clones were selected with puromycin for 14 days to establish the following new stable cell lines: SKOV3-NC, SKOV3-KD1, SKOV3-KD2, CAOV3-NC, CAOV3-KD1 and CAOV3-KD2.

MTT assay. Cell proliferation was assayed using a 3-(4,5-dimethylthiazol-2-yl)-2,5-diphenyltetrazolium bromide (MTT) kit (Sigma-Aldrich, St. Louis, MO, USA) and a Synergy H4 Hybrid Reader. Briefly, the culture medium was removed after $1,2,3,4$, and 5 days; $0.5 \mathrm{mg} / \mathrm{ml}$ MTT in $200 \mu \mathrm{l}$ of medium was added to each well; and the plates were incubated for $4 \mathrm{~h}$. Then, $150 \mu 1$ of DMSO was added to the SKOV3 and CAOV3 cells for $10 \mathrm{~min}$, and the optical density (OD) was measured at $490 \mathrm{~nm}$. Each experiment was repeated in triplicate.

Colony formation assay. Duplicate cultures of each cell type were maintained at $37^{\circ} \mathrm{C}$ in a $5 \% \mathrm{CO}_{2}$ atmosphere, and fresh medium was added every 3 days. After 2 weeks, colonies in each well consisting of $>50$ cells were counted. Each experiment was repeated in triplicate.

Tumour formation in nude mice. The animal experiments were approved by the Institutional Animal Care and Use Committee

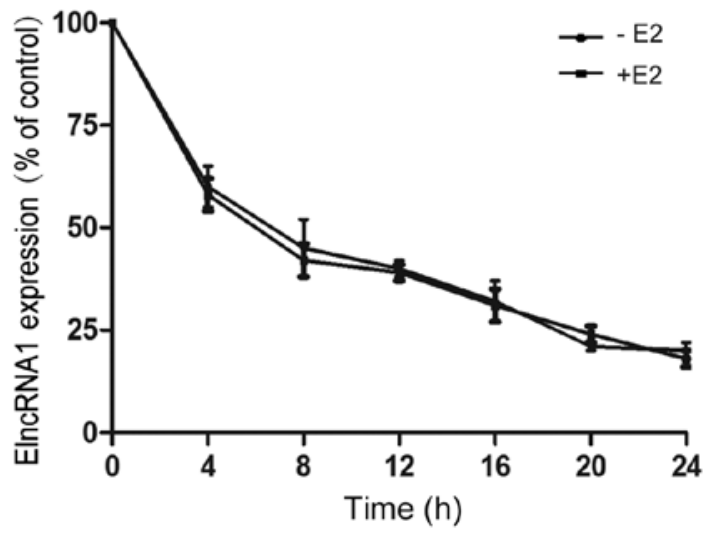

Figure 2. Effects of oestrogen on ElncRNA1 mRNA stability. The Y-axis shows relative ElncRNA1 expression levels in SKOV3 cells after incubation with actinomycin $\mathrm{D}$ for various times.

of Fudan University and were performed according to the institutional guidelines and protocols. SKOV3-KD1, SKOV3-KD2, and SKOV3-NC cells $\left(1 \times 10^{6}\right)$ were subcutaneously injected into 5-week-old BALB/c athymic nude mice (Department of Laboratory Animals, Fudan University; $n=7$ for each cell line). The mice were sacrificed after 4 weeks and examined for the growth of subcutaneous tumours. The tumour volume was calculated as previously described (33).

Western blotting. Western blot assays were performed using the following primary antibodies: anti-cyclin E (Santa Cruz); anti-cyclin D1 (Cell Signaling Technology); anti-CDK2 (Abways Technology); anti-CDK4 (Abways Technology); anti-CDK6 (Abways Technology); and $\beta$-actin (Proteintech). Briefly, stimulated cells were lysed with RIPA buffer [50 mM Tris-HCl (pH 7.5), $150 \mathrm{mM} \mathrm{NaCl}, 1 \%$ Triton X-100, 0.5\% Na-deoxycholate] containing protease inhibitors (Roche, Complete Mini); 20-30 $\mu \mathrm{g}$ samples of the lysates were separated on $8-12 \%$ SDS-PAGE gels and transferred to PVDF membranes. The membranes were incubated with primary antibodies overnight at $4^{\circ} \mathrm{C}$. The primary antibody incubation was followed by incubation with an HRP-conjugated secondary antibody. The bound antibodies were detected using an ECL kit (Pierce).

Statistical analysis. The data were processed using SPSS version 16.0 software (SPSS, Inc., Chicago, IL, USA). Continuous data were compared between the two groups using independent t-tests. P-values at $<0.05$ were considered statistically significant.

\section{Results}

A novel E2-induced lncRNA, TC0101441/ElncRNA1. We previously identified a novel lncRNA, TC0101441, which was significantly upregulated by E2 in SKOV3 ER $\alpha$-positive ovarian cancer cells (32). In this study, we designated TC0101441 as E2-induced lncRNA 1 (ElncRNA1). Based on a bioinformatics analysis using the UCSC genome browser (hg18, http:// genome.ucsc.edu/), we noted that ElncRNA1 is located on chromosome 1 (chr1: 202,377,159:202,378,011), contains two exons and encodes a 765 bp lncRNA molecule (Fig. 1). 


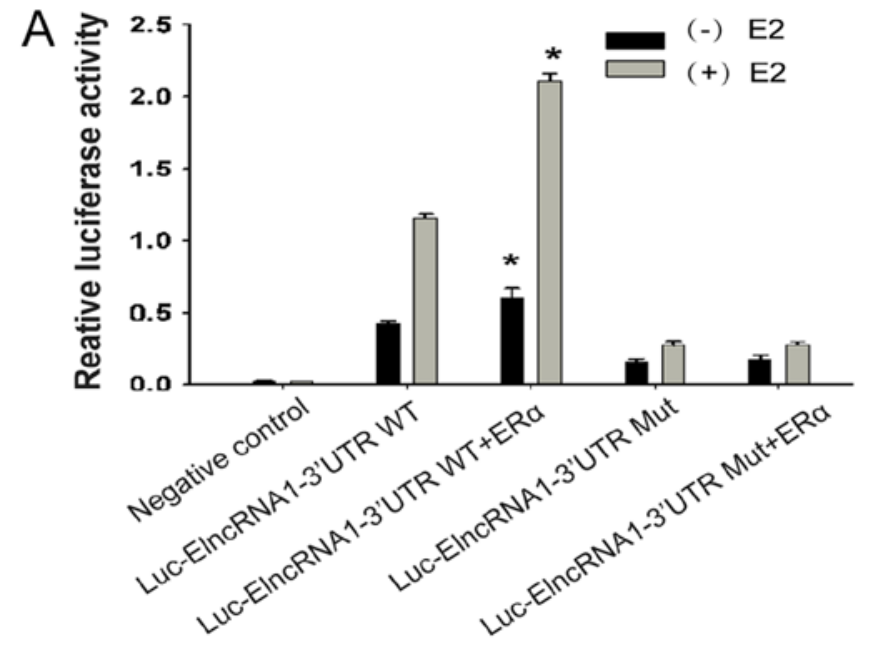

B

(-) E2

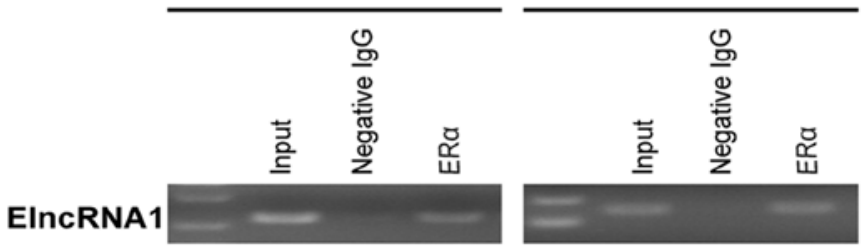

Figure 3.ER $\alpha$-ERE is required for the transcriptional regulation of ElncRNA1 by E2. (A) SKOV3 cells were transfected with ER $\alpha$ and ElncRNA1 promoter reporters containing a mutated or wild-type ERE. Luciferase activity was measured and normalized to Renilla luciferase activity. ${ }^{*} \mathrm{P}<0.05$. (B) ChIP analysis of ER $\alpha$ binding to the ElncRNA1 promoter. ChIP assays were performed with antibodies against $E R \alpha$ to confirm ER $\alpha$ binding to the ERE region within the ElncRNA1 promoter. Enrichment of ER $\alpha$-associated promoter fragments was confirmed by RT-PCR.

Effects of E2 on ElncRNA1 mRNA stability. To determine whether E2-mediated changes in ElncRNA1 levels are related to transcription or mRNA stability, we treated SKOV3 cells with actinomycin D to block nascent RNA synthesis prior to E2 treatment and measured ElncRNA1 levels at various subsequent time points. RNA stability was not significantly altered in SKOV3 cells in the absence or presence of E2 (Fig. 2). This result suggests that the E2-mediated changes in ElncRNA1 levels are not due to altered mRNA stability and implies that transcriptional regulation may be a major mechanism by which ElncRNA1 is induced.

ER $\alpha$-ERE binding is required for transcriptional regulation of ElncRNA1 expression by E2. Given the results of our prior study, which demonstrated that E2 induced ElncRNA1 expression in an ER $\alpha$-dependent manner (32), we next screened the ElncRNA1 promoter for the presence of ER $\alpha$-responsive elements to confirm the transcriptional upregulation of ElncRNA1 by E2. We identified a predicted ERE (-1725 to -1711) in a region 59 bp upstream of the TSS of ElncRNA1 (Fig. 1). We subsequently transfected SKOV3 cells with ER $\alpha$ and ElncRNA1 promoter reporters containing a mutated or wild-type (WT) ERE and then measured Luc activity after E2 treatment. Cells expressing both WT ERE and ER $\alpha$ responded strongly to E2 by inducing reporter activity (Fig. 3A), suggesting that both ER $\alpha$ and ERE are required for E2 to upregulate ElncRNA1 expression.

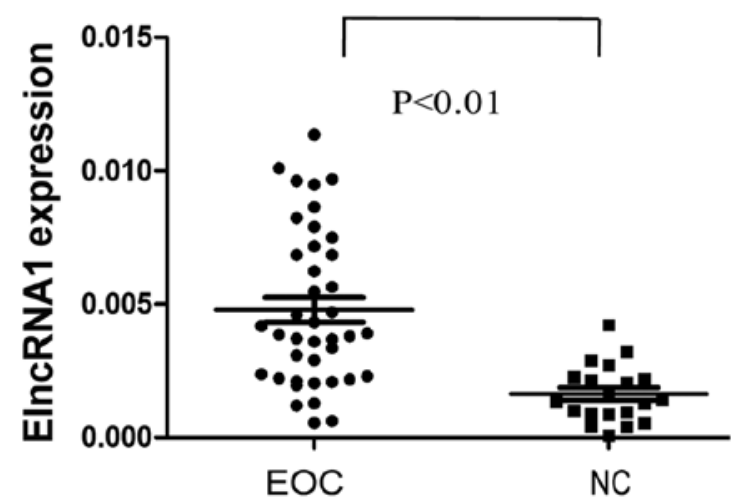

Figure 4. Relative ElncRNA1 expression in ovarian cancer tissues. ElncRNA1 expression in ovarian cancer tissues and normal ovarian tissues was determined by qRT-PCR.

We subsequently performed ChIP assays with antibodies against $\mathrm{ER} \alpha$ to confirm that $\mathrm{ER} \alpha$ binds to the ERE region of the ElncRNA1 promoter. As shown in Fig. 3B, enrichment of ER $\alpha$-associated promoter fragments was confirmed by RT-PCR, and this enrichment was enhanced by E2. These results indicate that ER $\alpha$ can bind to the ERE in the ElncRNA1 promoter in a manner that is enhanced by $\mathrm{E} 2$ treatment, thus inducing ElncRNA1 expression.

ElncRNAl expression pattern in EOC tissues. To investigate whether ElncRNA1 has clinical implications, we used qRT-PCR to determine the ElncRNA1 expression pattern in EOC. ElncRNA1 levels were significantly higher in 40 EOC tissues than in 20 normal ovarian surface epithelial tissues $(\mathrm{P}<0.01 ;$ Fig. 4). These results suggest that ElncRNA1 overexpression may play a role in EOC aggressiveness.

Silencing ElncRNA1 suppresses EOC cell proliferation in vitro. To determine whether ElncRNA1 affects EOC cell proliferation, we first examined the basal levels of ElncRNA1 in 4 EOC cell lines (SKOV3, CAOV3, OVCAR3 and HO8910) and found that SKOV3 and CAOV3 cells expressed relatively higher ElncRNA1 levels (Fig. 5A). Thus, we silenced ElncRNA1 expression in these two cell lines to investigate the effects of ElncRNA1 on cell proliferation. Since both siRNAs efficiently silenced ElncRNA1 expression in SKOV3 and CAOV3 cells (Fig. 5B), we constructed lentiviral vectors to establish stable ElncRNA1-knockdown cell lines (SKOV3-KD1, SKOV3-KD2, CAOV3-KD1, and CAOV3-KD2 cells) and corresponding controls (SKOV3-NC and CAOV3-NC cells).

MTT assays showed that ElncRNA1 knockdown significantly suppressed the proliferation of both SKOV3 and CAOV3 cells (Fig. 5C). Colony formation assays demonstrated that ElncRNA1 knockdown reduced the number of SKOV3 and CAOV3 colonies (Fig. 5D). These results indicate that silencing ElncRNA1 may inhibit EOC cell proliferation in vitro.

Silencing ElncRNA1 inhibits EOC tumour growth in vivo. Next, we tested the effects of ElncRNA1 on EOC tumour growth in vivo by injecting SKOV3-KD1, SKOV3-KD2 and SKOV3-NC cells into nude mice. ElncRNA1 knockdown reduced the frequency of tumour formation (SKOV3-NC, 7/7; 

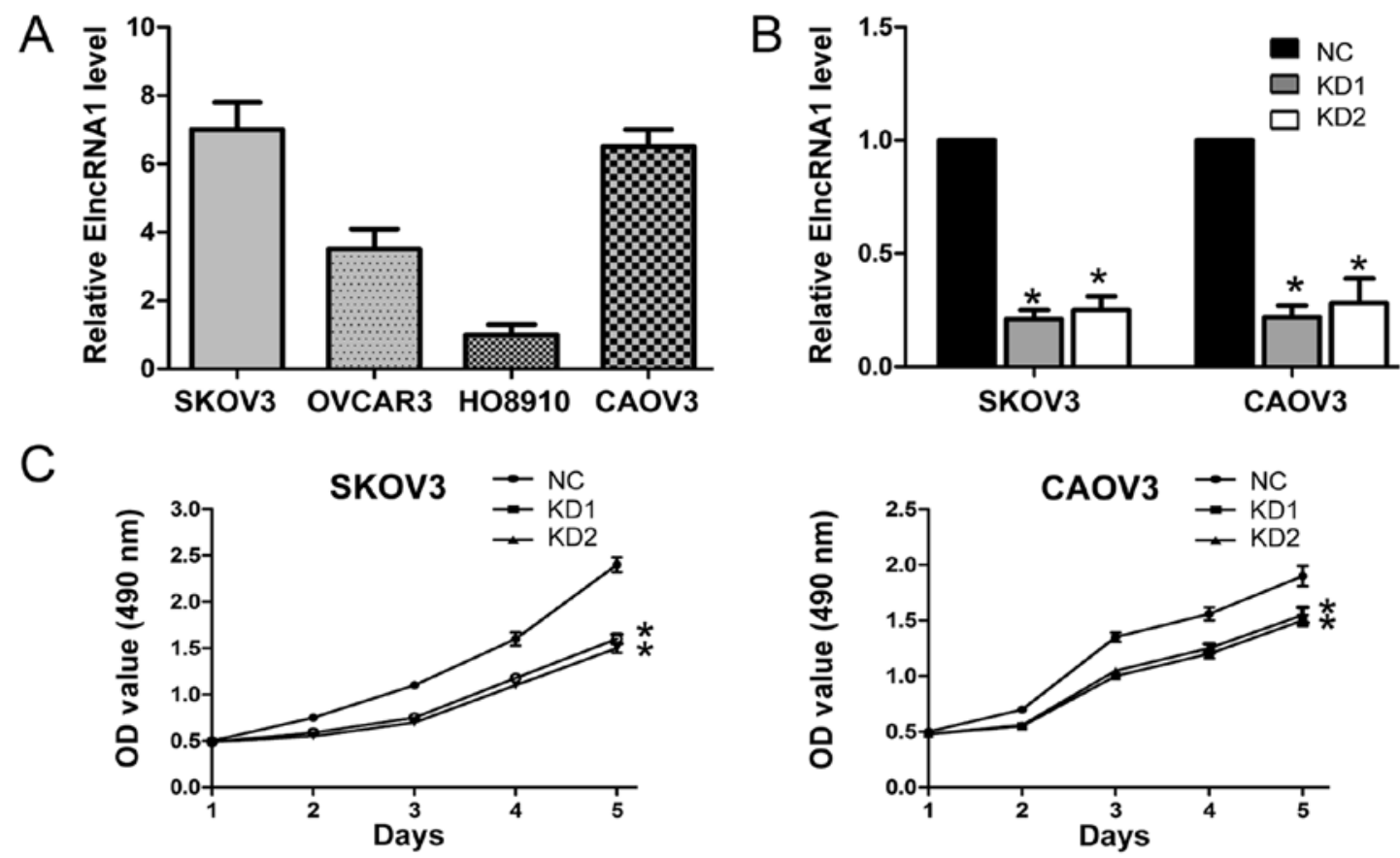

$\mathrm{D}$
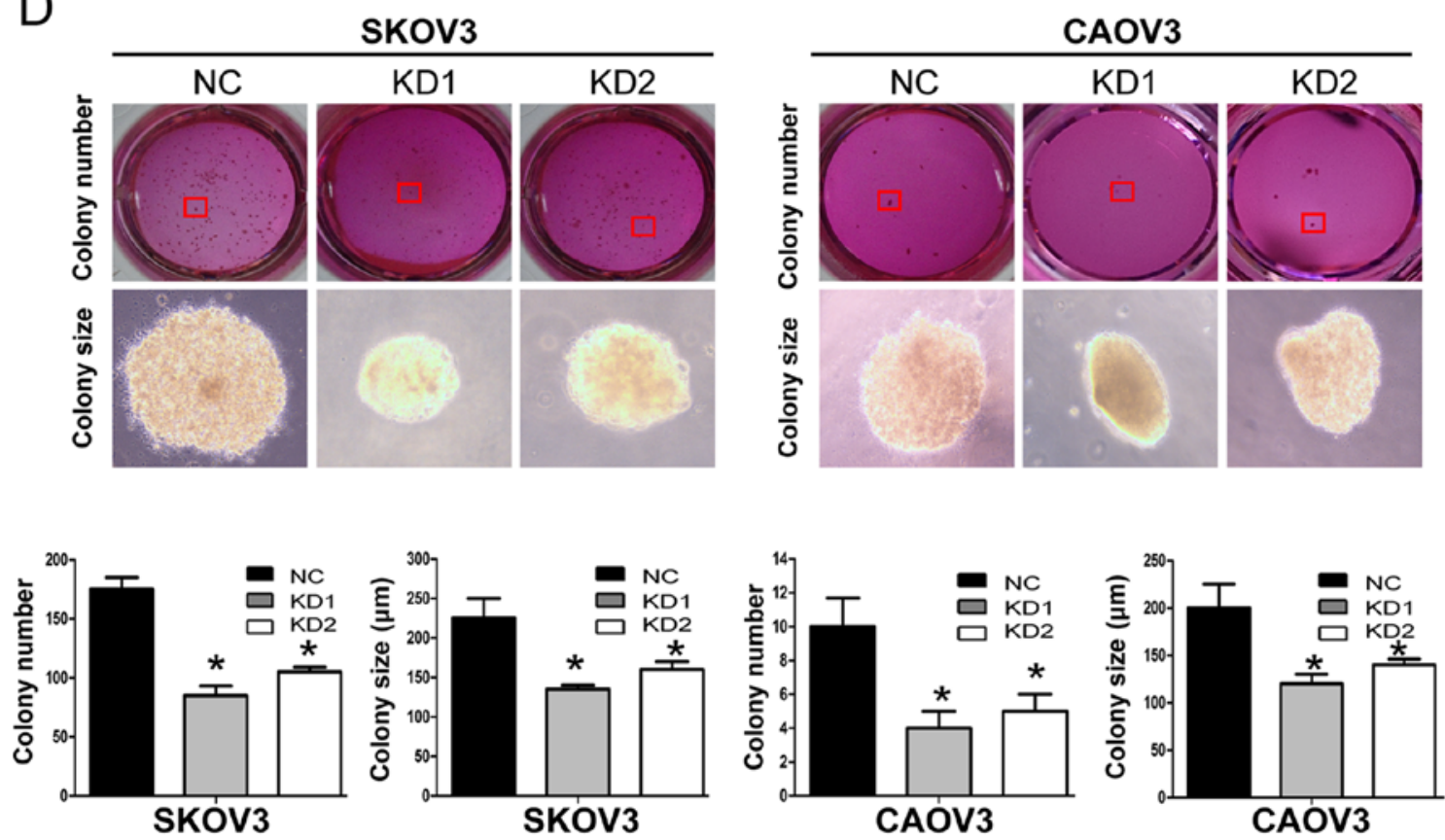

Figure 5. The effect of ElncRNA1 on ovarian cancer cell proliferation. (A) Relative ElncRNA1 expression levels in epithelial ovarian cancer cell lines (SKOV3, CAOV3, OVCAR3 and HO8910). (B) Relative ElncRNA1 expression levels in SKOV3 and CAOV3 cells transfected with si-NC or ElncRNA1-siRNAs. ${ }^{*} \mathrm{P}<0.05$. (C) MTT assays were performed to evaluate the proliferation of SKOV3-KD and CAOV3-KD cells compared with control cells. The data are presented as the mean \pm standard deviation (SD) of three independent experiments. The bars denote the SD. ${ }^{*} \mathrm{P}<0.05$. (D) Representative colony formation assay results, including colony number and size, for SKOV3-KD and CAOV3-KD cells and their corresponding controls. "P $<0.05$.

SKOV3-KD1, 4/7; and SKOV3-KD2,6/7) (Fig. 6A). Moreover, qRT-PCR analysis confirmed that ElncRNA1 expression levels were significantly lower in tumour tissues from the ElncRNA1-KD group relative to those from the control group (Fig. 6B). Further, the volume and average weight of tumours formed by SKOV3-KD1 and SKOV3-KD2 cells were much lower compared to tumours formed by SKOV3-NC cells (Fig. 6C). Together, these results suggest that silencing ElncRNA1 inhibits EOC growth in vivo, which corresponds with the in vitro results.
Certain proliferation-related proteins are downstream mediators of ElncRNA1 activity affecting EOC cell proliferation. To study possible mechanisms through which ElncRNA1 alters EOC cell proliferation further, we evaluated the expression of certain key proliferation-related proteins, including cyclin D1, cyclin E, CDK2, CDK4, CDK6, by western blot assay. The results showed that ElncRNA1 knockdown in SKOV3 and CAOV3 cells resulted in a significant decrease in CDK4, CDK6 and cyclin D1 protein levels (Fig. 7), suggesting that CDK4, CDK6 and cyclin D1 are the downstream media- 
A

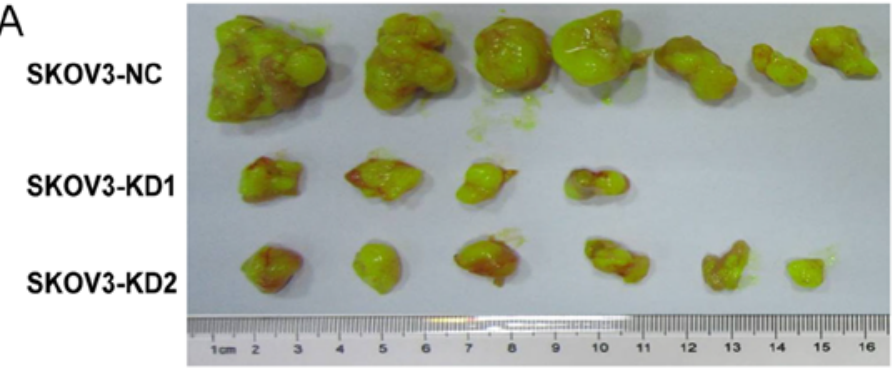

C

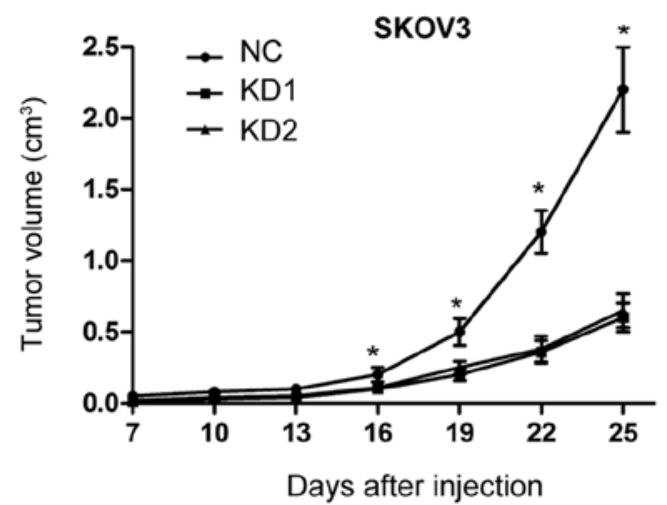

B

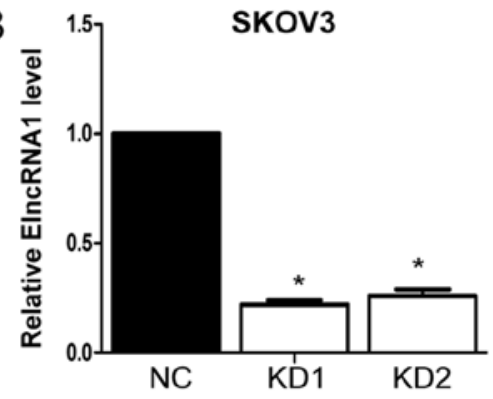

$\mathrm{D}$

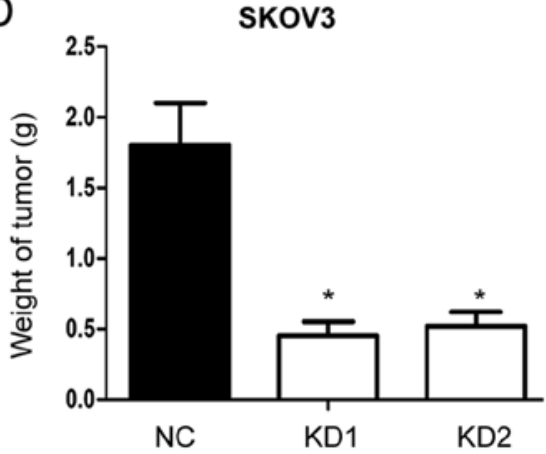

Figure 6. ElncRNA1 knockdown inhibits SKOV3 cell proliferation in vivo. (A) The frequency of tumour formation in the SKOV3-NC, SKOV3-KD1 and SKOV3-KD2 groups. (B) ElncRNA1 expression levels were lower in SKOV3-KD1 and SKOV3-KD2 tumours than in SKOV3-NC tumours. "P<0.05. (C) Growth curves of tumour xenografts. The volume of SKOV3-KD1 and SKOV3-KD2 tumours was substantially less than that of SKOV3-NC tumours, and the gap in tumour size between the KD groups and the NC group increased over time ("P<0.05). (D) SKOV3-NC tumours were significantly heavier than SKOV3-KD1 and SKOV3-KD2 tumours. "P<0.05.

tors of ElncRNA1 activity affecting EOC cell proliferation. Together, these data indicate that ElncRNA1 regulates EOC cell proliferation at least in part by regulating CDK4, CDK6 and cyclin D1.

\section{Discussion}

Previously, we identified the novel E2-upregulated lncRNA, ElncRNA1, based on microarray analysis. However, the detailed mechanisms by which E2 upregulates ElncRNA1 and the role of ElncRNA1 itself in EOC progression have not been determined (32). In the present study, using RNA stability assays, bioinformatics-based searches for ERE binding sites, ChIP assays and dual luciferase reporter assays, we found that E2 transcriptionally upregulated ElncRNA1 through the ER $\alpha$-ERE pathway. Clinically, ElncRNA1 levels were significantly higher in EOC tissues than in normal ovarian surface epithelial tissues. Further in vitro and in vivo assays revealed that ElncRNA1 promoted EOC cell proliferation. This proproliferation effect of ElncRNA1 was partially mediated by the regulation of CDK4, CDK6 and cyclin D1. Together, these findings not only clarify the mechanism by which E2 upregulates ElncRNA1 but also underscore the important role of this novel E2-upregulated IncRNA in EOC proliferation, thus providing a connection between E2 and ovarian cancer from the perspective of IncRNA.

E2 plays an important role in regulating cancer growth through various target genes, including protein coding genes and non-coding RNAs. We previously identified a novel E2-upregulated lncRNA (TC0101441, designated

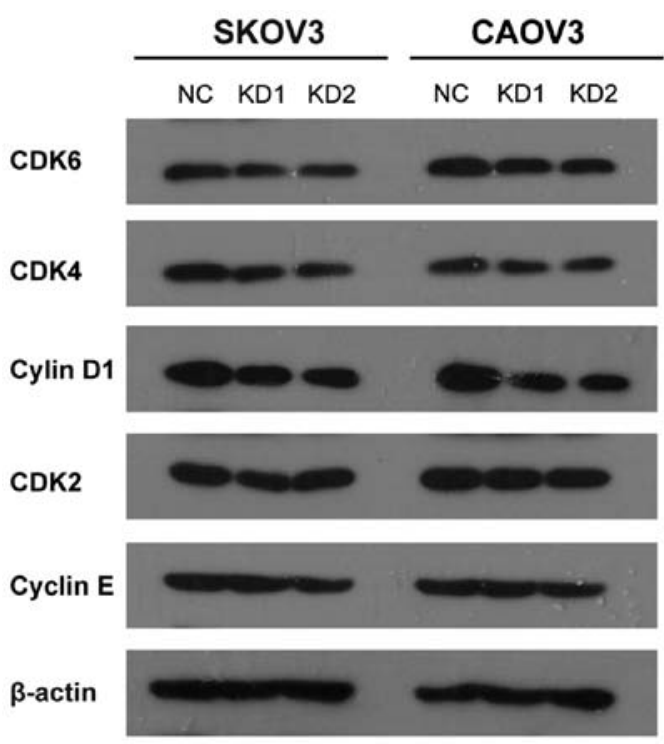

Figure 7. ElncRNA1 knockdown alters CDK4, CDK6 and cyclin D1 expression. Western blots showing that ElncRNA1 knockdown decreases CDK4, CDK6 and cyclin D1 expression levels in SKOV3 and CAOV3 cells.

ElncRNA1) (32), but the molecular mechanisms by which E2 upregulates ElncRNA1 have not been determined. So far, several mechanisms have been proposed for E2-regulated gene expression, including direct action of basal transcriptional elements and the participation of cofactors (34-36). Our prior study demonstrated that the E2-mediated induction of ElncRNA1 expression is dependent on ER $\alpha$ (32), a 
ligand-activated transcription factor in the nuclear receptor super-family that directly binds to oestrogen-responsive elements (EREs) in the promoter regions of target genes, thereby regulating their transcription $(34,36,37)$. Therefore, we hypothesized that ER $\alpha$-ERE binding might contribute to the transcriptional upregulation of ElncRNA1 expression by E2. To address this hypothesis, we initially performed mRNA stability experiments. The use of actinomycin D suggested that the E2-induced changes in ElncRNA1 expression were not due to effects on mRNA stability but were rather due to changes in E2-regulated transcription. To confirm the transcriptional upregulation of ElncRNA1 by E2, we screened the ElncRNA1 promoter and found an ERE in a 59 bp region upstream of the TSS of ElncRNA1. Subsequent ChIP assays indicated that $\operatorname{ER} \alpha$ bound to the ERE in the ElncRNA1 promoter. Moreover, dual luciferase reporter assays revealed that cells expressing both WT ERE and $E R \alpha$ responded strongly to $E 2$ by inducing reporter activity, suggesting that both $\operatorname{ER} \alpha$ and ERE are required for E2 to upregulate ElncRNA1 expression. Together, these findings indicate that E2 acts through its nuclear receptor $\mathrm{ER} \alpha$, which binds directly to ERE in the promoter region of ElncRNA1, thereby inducing transcriptional upregulation of ElncRNA1 expression. Additionally, we can conclude that E2 transcriptionally upregulates ElncRNA1 through the ER $\alpha$-ERE pathway.

However, the role of this novel E2-upregulated lncRNA, ElncRNA1, remained to be determined. Accumulating evidence indicates that aberrantly expressed lncRNAs play oncogenic or tumour suppressor roles in human cancer. For example, HOTAIR promotes tumour growth in cervical cancer (38), and MEG3 inhibits cell proliferation in prostate cancer (39). As IncRNAs are emerging as key components of cancer progression, it was reasonable to hypothesize that ElncRNA1 may contribute to EOC progression. Our previous work showed that ElncRNA1 contributes to E2-induced EOC metastasis. However, the role of ElncRNA1 itself in EOC has not been determined, and an association between ElncRNA1 and aspects of EOC progression other than metastasis has not been demonstrated. In the present study, ElncRNA1 levels were clearly higher in EOC tissues than in noncancerous tissues, suggesting that ElncRNA1 may play a role in the aggressiveness of EOC. In vitro, ElncRNA1 knockdown suppressed cell proliferation and reduced the number of SKOV3 and CAOV3 colonies. Additionally, in vivo experiments confirmed that ElncRNA1 depletion inhibited tumour growth in nude mice. Taken together, these data suggest that ElncRNA1 plays an important role in promoting EOC growth and can function as an oncogene, thereby contributing to the proliferative effects of E2.

Previous study demonstrated that the key proliferationrelated genes 'cyclin D1-CDK4/6', associated with E2/ER signaling pathway, promoted breast cancer proliferation (40). In the present study, we found that ElncRNA1 knockdown in SKOV3 and CAOV3 cells resulted in a significant decrease in CDK4, CDK6 and cyclin D1 protein levels instead of cyclin $\mathrm{E}$ and $\mathrm{CDK} 2$ levels, suggesting that ElncRNA1 regulates EOC cell proliferation at least in part by regulating 'cyclin D1-CDK4/6'. One limitation of this study is that we did not study the exact mechanism by which ElncRNA1 regulates cyclin D1-CDK4/6 in EOC growth. Further studies are required to explore the exact molecular mechanism of E2/ER $\alpha$ - ElncRNA1-'cyclin D1-CDK4/6' pathway.

In conclusion, our study provides the first evidence that E2 transcriptionally upregulates ElncRNA1 through the ER $\alpha$-ERE pathway and that this novel E2-upregulated lncRNA has an oncogenic role in EOC growth. The addition of ElncRNA1 to the E2-ER $\alpha$-ERE signalling pathway may provide greater insight into the oestrogenic effects on EOC progression from the perspective of IncRNA.

\section{Acknowledgements}

This study was supported by funding from the National Natural Science Foundation of China (81370689 and 81571404; to K.-Q.H.), the National Natural Science Foundation for Young Scholars of China (81502240; to J.-J.Q.), and the Shanghai Science and Technology Development Funds for the Talents (15YF1401400; to J.-J.Q.).

\section{References}

1. Hollis RL and Gourley C: Genetic and molecular changes in ovarian cancer. Cancer Biol Med 13: 236-247, 2016.

2. Doubeni CA, Doubeni AR and Myers AE: Diagnosis and management of ovarian cancer. Am Fam Physician 93: 937-944, 2016.

3. Siegel RL, Miller KD and Jemal A: Cancer statistics, 2016. CA Cancer J Clin 66: 7-30, 2016.

4. Tsilidis KK, Allen NE, Key TJ, Dossus L, Kaaks R, Bakken K, Lund E, Fournier A, Dahm CC, Overvad K, et al: Menopausal hormone therapy and risk of ovarian cancer in the European prospective investigation into cancer and nutrition. Cancer Causes Control 22: 1075-1084, 2011.

5. Cunat S, Hoffmann P and Pujol P: Estrogens and epithelial ovarian cancer. Gynecol Oncol 94: 25-32, 2004.

6. Park SH, Cheung LW, Wong AS and Leung PC: Estrogen regulates Snail and Slug in the down-regulation of E-cadherin and induces metastatic potential of ovarian cancer cells through estrogen receptor alpha. Mol Endocrinol 22: 2085-2098, 2008.

7. Hua K, Din J, Cao Q, Feng W, Zhang Y, Yao L, Huang Y, Zhao Y and Feng Y: Estrogen and progestin regulate HIF-1 $\alpha$ expression in ovarian cancer cell lines via the activation of Akt signaling transduction pathway. Oncol Rep 21: 893-898, 2009.

8. Hua K, Feng W, Cao Q, Zhou X, Lu X and Feng Y: Estrogen and progestin regulate metastasis through the PI3K/AKT pathway in human ovarian cancer. Int J Oncol 33: 959-967, 2008.

9. Ding JX, Feng YJ, Yao LQ, Yu M, Jin HY and Yin LH: The reinforcement of invasion in epithelial ovarian cancer cells by 17 beta-Estradiol is associated with up-regulation of Snail. Gynecol Oncol 103: 623-630, 2006.

10. Spillman MA, Manning NG, Dye WW, Sartorius CA, Post MD, Harrell JC, Jacobsen BM and Horwitz KB: Tissue-specific pathways for estrogen regulation of ovarian cancer growth and metastasis. Cancer Res 70: 8927-8936, 2010.

11. Laviolette LA, Garson K, Macdonald EA, Senterman MK, Courville K, Crane CA and Vanderhyden BC: 17beta-estradiol accelerates tumor onset and decreases survival in a transgenic mouse model of ovarian cancer. Endocrinology 151: 929-938, 2010.

12. O'Donnell AJ, Macleod KG, Burns DJ, Smyth JF and Langdon SP: Estrogen receptor-alpha mediates gene expression changes and growth response in ovarian cancer cells exposed to estrogen. Endocr Relat Cancer 12: 851-866, 2005.

13. Gutschner T, Hämmerle M, Eissmann M, Hsu J, Kim Y, Hung G, Revenko A, Arun G, Stentrup M, Gross M, et al: The noncoding RNA MALAT1 is a critical regulator of the metastasis phenotype of lung cancer cells. Cancer Res 73: 1180-1189, 2013.

14. Du Y, Kong G, You X, Zhang S, Zhang T, Gao Y, Ye L and Zhang X: Elevation of highly up-regulated in liver cancer (HULC) by hepatitis B virus $\mathrm{X}$ protein promotes hepatoma cell proliferation via down-regulating p18. J Biol Chem 287: 26302-26311, 2012. 
15. Gupta RA, Shah N, Wang KC, Kim J, Horlings HM, Wong DJ, Tsai MC, Hung T, Argani P, Rinn JL, et al: Long non-coding RNA HOTAIR reprograms chromatin state to promote cancer metastasis. Nature 464: 1071-1076, 2010.

16. Matouk IJ, DeGroot N, Mezan S, Ayesh S, Abu-lail R, Hochberg A and Galun E: The H19 non-coding RNA is essential for human tumor growth. PLoS One 2: e845, 2007.

17. Huarte M, Guttman M, Feldser D, Garber M, Koziol MJ, Kenzelmann-Broz D, Khalil AM, Zuk O, Amit I, Rabani M, et al: A large intergenic noncoding RNA induced by p53 mediates global gene repression in the p53 response. Cell 142: 409-419, 2010.

18. Zhou Y, Zhang X and Klibanski A: MEG3 noncoding RNA: A tumor suppressor. J Mol Endocrinol 48: R45-R53, 2012.

19. Qiao HP, Gao WS, Huo JX and Yang ZS: Long non-coding RNA GAS5 functions as a tumor suppressor in renal cell carcinoma. Asian Pac J Cancer Prev 14: 1077-1082, 2013.

20. Fang J, Sun CC and Gong C: Long noncoding RNA XIST acts as an oncogene in non-small cell lung cancer by epigenetically repressing KLF2 expression. Biochem Biophys Res Commun 478: 811-817, 2016.

21. Li J, Yu H, Xi M and Lu X: Long noncoding RNA C17orf91 is a potential prognostic marker and functions as an oncogene in ovarian cancer. J Ovarian Res 9: 49, 2016

22. Liao T, Qu N, Shi RL, Guo K, Ma B, Cao YM, Xiang J, Lu ZW, Zhu YX, Li DS, et al: BRAF-activated LncRNA functions as a tumor suppressor in papillary thyroid cancer. Oncotarget 8: 238-247, 2017.

23. Sang Y, Zhou F, Wang D, Bi X, Liu X, Hao Z, Li Q and Zhang W: Up-regulation of long non-coding HOTTIP functions as an oncogene by regulating HOXA13 in non-small cell lung cancer. Am J Transl Res 8: 2022-2032, 2016.

24. Tanos V, Prus D, Ayesh S, Weinstein D, Tykocinski ML, De-Groot N, Hochberg A and Ariel I: Expression of the imprinted H19 oncofetal RNA in epithelial ovarian cancer. Eur J Obstet Gynecol Reprod Biol 85: 7-11, 1999.

25. Rangel LB, Sherman-Baust CA, Wernyj RP, Schwartz DR, Cho KR and Morin PJ: Characterization of novel human ovarian cancer-specific transcripts (HOSTs) identified by serial analysis of gene expression. Oncogene 22: 7225-7232, 2003.

26. Silva JM, Boczek NJ, Berres MW, Ma X and Smith DI: LSINCT5 is over expressed in breast and ovarian cancer and affects cellular proliferation. RNA Biol 8: 496-505, 2011.

27. Qiu JJ, Wang Y, Liu YL, Zhang Y, Ding JX and Hua KQ: The long non-coding RNA ANRIL promotes proliferation and cell cycle progression and inhibits apoptosis and senescence in epithelial ovarian cancer. Oncotarget 7: 32478-32492, 2016.

28. Qiu JJ, Lin YY, Ye LC, Ding JX, Feng WW, Jin HY, Zhang Y, Li Q and Hua KQ: Overexpression of long non-coding RNA HOTAIR predicts poor patient prognosis and promotes tumor metastasis in epithelial ovarian cancer. Gynecol Oncol 134: $121-128,2014$
29. Chooniedass-Kothari S, Vincett D, Yan Y, Cooper C, Hamedani MK, Myal Y and Leygue E: The protein encoded by the functional steroid receptor RNA activator is a new modulator of ER alpha transcriptional activity. FEBS Lett 584: 1174-1180, 2010.

30. Bhan A and Mandal SS: Estradiol-induced transcriptional regulation of Long non-coding RNA, HOTAIR. Methods Mol Biol 1366: 395-412, 2016.

31. Xue X, Yang YA, Zhang A, Fong KW, Kim J, Song B, Li S, Zhao JC and Yu J: LncRNA HOTAIR enhances ER signaling and confers tamoxifen resistance in breast cancer. Oncogene 35: 2746-2755, 2016.

32. Qiu J, Ye L, Ding J, Feng W, Zhang Y, Lv T, Wang J and Hua K: Effects of oestrogen on long noncoding RNA expression in oestrogen receptor alpha-positive ovarian cancer cells. J Steroid Biochem Mol Biol 141: 60-70, 2014.

33. Wang Y, Wang Z, Qi Z, Yin S, Zhang N, Liu Y, Liu M, Meng J, Zang R, Zhang Z, et al: The negative interplay between Aurora $\mathrm{A} / \mathrm{B}$ and $\mathrm{BRCA} 1 / 2$ controls cancer cell growth and tumorigenesis via distinct regulation of cell cycle progression, cytokinesis, and tetraploidy. Mol Cancer 13: 94, 2014.

34. Lin CY, Vega VB, Thomsen JS, Zhang T, Kong SL, Xie M, Chiu KP, Lipovich L, Barnett DH, Stossi F, et al: Whole-genome cartography of estrogen receptor alpha binding sites. PLoS Genet 3: e87, 2007.

35. Kushner PJ, Agard DA, Greene GL, Scanlan TS, Shiau AK, Uht RM and Webb P: Estrogen receptor pathways to AP-1. J Steroid Biochem Mol Biol 74: 311-317, 2000.

36. Carroll JS, Meyer CA, Song J, Li W, Geistlinger TR, Eeckhoute J, Brodsky AS, Keeton EK, Fertuck KC, Hall GF, et al: Genome-wide analysis of estrogen receptor binding sites. Nat Genet 38: 1289-1297, 2006.

37. Huang Y, Li X and Muyan M: Estrogen receptors similarly mediate the effects of $17 \beta$-estradiol on cellular responses but differ in their potencies. Endocrine 39: 48-61, 2011.

38. Lee M, Kim HJ, Kim SW, Park SA, Chun KH, Cho NH, Song YS and Kim YT: The long non-coding RNA HOTAIR increases tumour growth and invasion in cervical cancer by targeting the Notch pathway. Oncotarget 7: 44558-44571, 2016.

39. Luo G, Wang M, Wu X, Tao D, Xiao X, Wang L, Min F, Zeng F and Jiang G: Long non-coding RNA MEG3 inhibits cell proliferation and induces apoptosis in prostate cancer. Cell Physiol Biochem 37: 2209-2220, 2015.

40. Herrera-Abreu MT, Palafox M, Asghar U, Rivas MA, Cutts RJ, Garcia-Murillas I, Pearson A, Guzman M, Rodriguez O, Grueso J, et al: Early adaptation and acquired resistance to CDK4/6 inhibition in estrogen receptor-positive breast cancer. Cancer Res 76: 2301-2313, 2016. 